\title{
BONE LOSS RECOVERY IN MICE FOLLOWING MICROGRAVITY WITH CONCURRENT BONE-COMPARTMENT-SPECIFIC OSTEOCYTE CHARACTERISTICS
}

\author{
S. von Kroge ${ }^{1, \$}$, E.M. Wölfel ${ }^{1,2, \$}$, L.B. Buravkova ${ }^{3}$, D.A. Atiakshinn ${ }^{4,5}$, E.A. Markina ${ }^{3}$, T. Schinke ${ }^{1}$, T. Rolvien ${ }^{1,6}$,
} B. Busse ${ }^{1,2}$ and K. Jähn-Rickert ${ }^{1,7, *}$

\author{
${ }^{1}$ Department of Osteology and Biomechanics, University Medical Centre Hamburg-Eppendorf, Hamburg, Germany \\ ${ }^{2}$ Interdisciplinary Competence Centre for Interface Research (ICCIR), Forum Medical Technology Health \\ Hamburg (FMTHH), Hamburg, Germany \\ ${ }^{3}$ Institute of Biomedical Problems of RAS, State Scientific Centre of Russian Federation, Moscow, Russia \\ ${ }^{4}$ Research and Educational Resource Center for Immunophenotyping, Digital Spatial Profiling and \\ Ultrastructural Analysis Innovative Technologies, Peoples' Friendship University of Russia, Moscow, \\ Russia \\ ${ }^{5}$ Institute of Experimental Biology and Medicine, Voronezh State Medical University named after N.N. \\ Burdenko, Voronezh, Russia \\ ${ }^{6}$ Department of Trauma and Orthopaedic Surgery, Division of Orthopaedics, \\ University Medical Centre Hamburg-Eppendorf, Hamburg, Germany \\ ${ }^{7}$ Mildred Scheel Cancer Career Centre Hamburg, University Cancer Centre Hamburg, \\ University Medical Centre Hamburg-Eppendorf, Hamburg, Germany \\ $\S$ These authors contributed equally
}

\begin{abstract}
Space missions provide the opportunity to investigate the influence of gravity on the dynamic remodelling processes in bone. Mice were examined following space flight and subsequent recovery to determine the effects on bone compartment-specific microstructure and composition. The resulting bone loss following microgravity recovered only in trabecular bone, while in cortical bone the tissue mineral density was restored after only one week on Earth. Detection of TRAP-positive bone surface cells in the trabecular compartment indicated increased resorption following space flight. In cortical bone, a persistent reduced viability of osteocytes suggested an impaired sensitivity to mechanical stresses. A compartment-dependent structural recovery from microgravity-induced bone loss was shown, with a direct osteocytic contribution to persistent low bone volume in the cortical region even after a recovery period. Trabecular recovery was not accompanied by changes in osteocyte characteristics. These post-space-flight findings will contribute to the understanding of compositional changes that compromise bone quality caused by unloading, immobilisation, or disuse.
\end{abstract}

Keywords: Microgravity, mineralisation, bone compartments, osteocyte, bone remodelling.

*Address for correspondence: K. Jähn-Rickert PhD, Department of Osteology and Biomechanics, University Medical Centre Hamburg-Eppendorf, Lottestrasse 55a, 22529 Hamburg, Germany.

Telephone number: +49 40741026301 Email: k.jaehn@uke.de

Copyright policy: This article is distributed in accordance with Creative Commons Attribution Licence (http://creativecommons.org/licenses/by-sa/4.0/).

\begin{tabular}{|c|c|c|c|}
\hline & List of Abbreviations & $\begin{array}{l}\mathrm{Ct} \\
\mathrm{DXA}\end{array}$ & $\begin{array}{l}\text { cortical } \\
\text { dual energy x-ray absorptiometry }\end{array}$ \\
\hline $2 \mathrm{D}$ & 2 dimensional & e & empty \\
\hline 3D & 3 dimensional & G & on the ground \\
\hline aBMD & areal bone mineral density & $\mathrm{G}_{\mathrm{rec}}$ & on the ground following recovery \\
\hline BMDD & bone mineral density distribution & & period \\
\hline $\mathrm{BPm}$ & bone perimeter & ID & inner diameter \\
\hline BS & bone surface & IQR & interquartile range \\
\hline BV & bone volume & Lc & lacuna \\
\hline CaMean & mean calcium content & $\mathrm{LCN}$ & lacuno-canalicular network \\
\hline Cell-S & cellular surface & MCT & mean cortex thickness \\
\hline
\end{tabular}




$\begin{array}{ll}\text { N } & \text { number } \\ \text { OD } & \text { outer diameter } \\ \text { Ot } & \text { osteocyte } \\ \text { PMMA } & \text { polymethylmethacrylate } \\ \text { qBEI } & \begin{array}{l}\text { quantitative backscattered electron } \\ \text { imaging } \\ \text { receptor activator of nuclear factor } \\ \text { kappa-B ligand }\end{array} \\ & \text { in space group } \\ \text { S } & \text { in space group following recovery } \\ S_{\text {rec }} & \text { period } \\ \text { Sp } & \text { spacing } \\ \text { Tb } & \text { trabecular } \\ \text { Th } & \text { thickness } \\ \text { TMD } & \text { tissue mineral density } \\ \text { TRAP } & \text { tartrate-resistant acid phosphatase } \\ \text { TUNEL } & \text { terminal deoxynucleotidyl transferase } \\ & \text { dUTP nick end labeling } \\ \text { TV } & \text { tissue volume } \\ \text { V } & \text { volume } \\ \text { Wnt } & \text { Wingless and Int-1 } \\ \mu C T & \text { micro-computed tomography }\end{array}$

\section{Introduction}

Missions to space have long been a dream of mankind. However, the burden they impose on the human body is known since the first missions in 1961-63 (Smith et al., 2014). On Earth, a constant gravitational force acts on the human body. In space, microgravity-induced weightlessness causes detrimental effects on all tissues, including the musculoskeletal system. These are caused by the effects of large forces during takeoff and landing, radiation exposure, reduced exercise, and limited social interactions, as well as the loss of the light-induced circadian rhythm (Buravkova et al., 2010; Collet et al. 1997; Vico et al., 2000). Bone, as an essential biomaterial assuring mobility, protection, and a mineral stock in a healthy adult, e.g. a candidate astronaut, is constantly remodelled by the activity of matrix-building and mineralising osteoblasts as well as resorbing osteoclasts. Space missions lead to weight loss, contributed to by a rapid loss of muscle mass and a significantly reduced bone volume (Caillot-Augusseau et al., 1998; Grimm et al., 2016; Stein 2013; Vico et al., 2000). It is important to either prevent such changes, or allow post-mission recovery; however, understanding their mechanistics could aid bone research on Earth, e.g. for the treatment of immobilisation-induced bone loss.

The nano-composition of bone is constantly being remodelled and has been shown to be imbalanced, favouring increased bone resorption with unchanged or reduced bone formation and a rapid decrease in bone-matrix-stored minerals during space missions. This is also observed following prolonged periods of immobilisation (Smith et al., 1999). Reduced bonemineral content during space missions is site-specific, occurring predominantly in load-bearing bones (Orwoll et al., 2013; Vico et al., 2000). This suggests that unloading of skeletal regions to be the main cause of bone mineral loss. Reports on readaptation following unloading or microgravity indicate at least a $2.5 \times$ longer recovery period for bone mass, which could be dependent on mission duration, skeletal site, and bone compartment (Hargens and Vico 2016; Leblanc et al., 1990; Orwoll et al., 2013; Rolvien et al., 2020; Smith et al., 1999; Sotnezova et al., 2017).

Residing in the bone matrix, osteocytes - which are terminally differentiated osteoblasts - orchestrate the coupling of bone remodelling. By forming an interconnected fluid-filled network through the LCN, osteocytes allow mechanosensitivity within the dense bone matrix and communication to surface cells. Specifically, simulated microgravity induces osteocyte expression of the Wnt inhibitor sclerostin, repressing bone formation by osteoblasts, and of the RANKL, activating bone-resorbing osteoclasts (Spatz et al., 2015). Additionally, osteocytes directly contribute to mineral homeostasis by osteocytic osteolysis in situations of extreme calcium demand, e.g. during lactation (Jähn et al. 2017) or vitamin D deficiency (Rolvien et al., 2017). Bone resorption by osteocytes under microgravity is not consistently reported and may depend upon a variety of factors, including which bone compartment is studied (Blaber et al., 2013; Gerbaix et al., 2017). In addition, osteocyte viability and network connectivity are the basis of functional mechano-regulated bone matrix turnover. Osteocyte apoptosis was evident in a murine model of immobilisation using tail suspension (Aguirre et al., 2006) and has been suggested by some, but not all, studies to accompany microgravity-induced bone loss (Blaber et al., 2013; Gerbaix et al., 2017). Recently, lower numbers of osteocyte canaliculi in human Ct-bone from immobilised individuals were reported (Rolvien et al., 2020), suggesting impaired lacuno-canalicular characteristics due to mechanical unloading, which remains to be determined regarding microgravity.

The first studies of the impact of space missions on osteocytes were performed; however, controversial data have been found regarding osteocyte viability and osteocytic osteolysis (Blaber et al., 2013). With Ct-bone being metabolically less active while participating in load bearing and fracture resistance, $\mathrm{Tb}$-bone is more often subject to bone remodelling (Allen and Burr, 2014), suggesting that post space mission differential changes would be expected in these bone compartments. For better preventive strategies during space flight or long-term immobilisation, an understanding of compartmentspecific bone responses - including determining osteocyte characteristics - are needed during and following space missions in order to understand the influence of gravitational forces on bone quality.

In this study, the tibiae of mice that went on the Russian BION-M1 mission in 2013 were analysed to determine the effects of a one-month space flight and a $7 \mathrm{~d}$ recovery on Earth on $\mathrm{Tb}$ and $\mathrm{Ct}$-bone. Specifically, a multiscale nano- 
composite characterisation, including $\mu \mathrm{CT}$, qBEI, immunohistochemistry, and histology were performed to evaluate compartment-specific changes in bone microstructure, mineralisation and osteocyte viability and activity during and following space missions. Previous studies suggest that, in addition to a reduced amount of bone, microgravity might induce long-term alterations within the bone matrix (Sibonga et al., 2007; Vico et al., 2000). Therefore, the working hypothesis was that a distinct response of $\mathrm{Tb}$ - and Ct-bone during recovery - following space flight - might lead to compartment-specific changes in bone quality. Furthermore, compartment-specific negative effects of unloading on osteocyte viability and canalicular connections, impacting osteocyte regulation of bone remodelling following space flight are suggested.

\section{Materials and Methods}

\section{Experimental design}

Male wild-type C57BL/6N mice were obtained from the animal nursery of the Shemyakina-Ovchinnikov Institute of Bioorganic Chemistry (Moscow, Russia) at the age of 8-9 weeks, with specified pathogenfree status. As pre-adaptation for flight and ground experiments, mice were split into small groups of 3 animals and kept in $1.7 \mathrm{~L}$ cylindrical habitats on a $12 \mathrm{~h} / 12 \mathrm{~h}$ day/night cycle, mimicking experimental conditions. Two weeks prior to the start of the experiment, the regular diet was changed to a special flight-paste food - based on pelleted food, water and casein - that was provided at a daily dosage of $54 \mathrm{~g}$ per 3 mice. The habitats ensured the same climatic conditions for all mice throughout both flight and ground experiments. More detailed information on selection process, housing and feeding conditions are described elsewhere (Andreev-Andrievskiy et al., 2014). For the flight and ground experiments, animal housing groups were randomly divided into 4 experimental groups. Two groups were sent to space on the BION-M1 biosatellite mission for 4 weeks and subsequently sacrificed $(S, n=5)$ or kept on the ground for $7 \mathrm{~d}$ to recover post space mission $\left(\mathrm{S}_{\mathrm{rec}^{\prime}} n=5\right)$. A recovery period of $7 \mathrm{~d}$ was chosen in order to determine bone-related changes directly following a space flight. This was done to provide new information and create evidence for optimal treatment strategies following space flight. The apposition rate and bone remodelling cycle in 3 months old C57BL/6N mice are documented as $2 \mu \mathrm{m} / \mathrm{d}$ and 2 weeks, respectively (Allen \& Burr, 2014; Jilka, 2013). This suggests detection of the first potential bone formation after $7 \mathrm{~d}$ of re-ambulation. As controls, 2 groups of mice were housed on the ground for 4 weeks $(\mathrm{G}, n=6)$ and 5 weeks $\left(\mathrm{G}_{\text {rec }}\right.$ $n=6)$. Over the course of the experiments, a similar locomotive behaviour - with frequent animal aggregations at the habitat walls - was observed in all groups. The differences in bodyweight between space-flight and ground-control groups were not significant (Andreev-Andrievskiy et al., 2014). A bioethical examination of the study protocol was carried out by the Bioethics Commission of the Research Institute of Mitoengineering of Moscow State University (protocol \# 35 of November 1, 2012) and the Commission on Biomedical Ethics of the State Scientific Centre of the Russian Federation - Institute of Biomedical Problems of the Russian Academy of Sciences (protocol \# 319 of April 4, 2013) (Andreev-Andrievsky et al., 2014). The experiments were carried out in accordance with the European Convention for the Protection of Vertebrate Animals Used for Experiments or for Other Scientific Purposes (Strasbourg, March 18, 1986) and order \# 742 of the USSR Ministry of Higher and Secondary Special Education "on approving the rules for work with the use of experimental animals" from 13.11.1984.

\section{Skeletal microstructure}

Following the space mission, $9 \mathrm{~h}$ post landing, and recovery time, the tibiae of the mice were dissected and frozen in the laboratory of the Institute of Biomedical Problems, Moscow, Russia. All skeletal analyses were performed at the Institute of Osteology and Biomechanics, UKE, Hamburg, Germany. Tibiae were fixed in $3.5 \%$ neutral buffered formalin and scanned using a $\mu \mathrm{CT}(\mu \mathrm{CT} 40$, Scanco Medical AG, Switzerland) at $55 \mathrm{kV}, 145 \mu \mathrm{A}$, a voxel size of $10 \mu \mathrm{m}$, and an integration time of $200 \mathrm{~ms}$. Three-dimensional reconstruction was evaluated using the software provided by the manufacturer to assess $\mathrm{Tb}-\mathrm{BV} / \mathrm{TV}$ (\%), Tb-Th (mm), Tb-N (1/mm), Tb-Sp (mm), and BS/ $\mathrm{BV}(\mathrm{mm})$. Ct parameters assessed were Ct-Th $(\mathrm{mm})$, $\mathrm{Ct}-\mathrm{BS} / \mathrm{BV}(1 / \mathrm{mm})$, and TMD $\left(\mathrm{mgHA} / \mathrm{cm}^{3}\right) . \mu \mathrm{CT}$ image slides were used to determine the OD ( $\mathrm{mm})$, ID (mm), and MCT (OD-ID/2, mm) using the interosseous crest as an anatomical landmark to measure the thickness of the cortex on both sides divided by 2 in the medial lateral direction.

In accordance with international guidelines (Bouxsein et al., 2010), the $\mathrm{Tb}$ microstructure at $0.5 \mathrm{~mm}$ to $1.5 \mathrm{~mm}$ distal to the growth plate was assessed, avoiding the primary spongiosa. The $\mathrm{Ct}$ microstructure was determined at $1 \mathrm{~mm}$ to $1.5 \mathrm{~mm}$ proximal to the tibia-fibula fusion landmark.

\section{BMDD}

Tibiae were embedded into PMMA and cut with a microtome (Leica Microsystems, Wetzlar, Germany) to obtain $4 \mu \mathrm{m}$ bone sections for histological assessment (Zimmermann et al., 2015). qBEI using a scanning electron microscope (LEO $435 \mathrm{VP}$; LEO Electron Microscopy Ltd., Cambridge, UK) with a backscattered electron detector (Type 202; K.E. Developments Ltd., Cambridge, UK) was performed at $20 \mathrm{kV}, 680 \mathrm{pA}$, and with a constant working distance of $20 \mathrm{~mm}$ on the longitudinal midsection and proximal spongiosa as previously described (Busse 
et al. 2009; Rolvien et al. 2019). With a customised MATLAB routine (MATLAB R2014a, MathWorks ${ }^{\circledR}$, MA, USA), the mean calcium content (CaMean, wt \%) in the proximal region for $\mathrm{Tb}$ and in the midshaft region for $\mathrm{Ct}$-bone was determined.

Tb-TMD and osteocyte lacunar analysis

Embedded proximal tibiae were further scanned using $\mathrm{CCT}$ (Skyscan 1272, Bruker, Kontich, Belgium) at a voxel size of $0.8 \mu \mathrm{m}, 80 \mathrm{kV}$ accelerating voltage, $124 \mu \mathrm{A}$ tube current, 3,800 ms exposure time, and $0.2^{\circ}$ rotation step to three-dimensionally characterise both Tb-TMD (mgHA/ccm) by precalibration using hydroxyapatite phantoms and $\mathrm{Tb}$ and $\mathrm{Ct}$ osteocyte lacunar morphology. The region of interest was defined from 0.5 to $1.5 \mathrm{~mm}$ distal to the growth zone to avoid the inclusion of primary spongiosa, and postprocessing was performed as described previously by Hemmatian et al. (2017) to determine the lacuna number density (N-Lc/TV) and the mean Lc-V. In detail, a global grey value threshold, beamhardening correction and ring-artifact reduction was applied to all samples and evaluated automatically using CTAn software (Bruker, Kontich, Belgium). A histogram-based global threshold was applied to segment mineralised from non-mineralised tissue. Images were inverted using the 3D despeckle filter and analysed using Individual Object Analysis

\section{Study design}
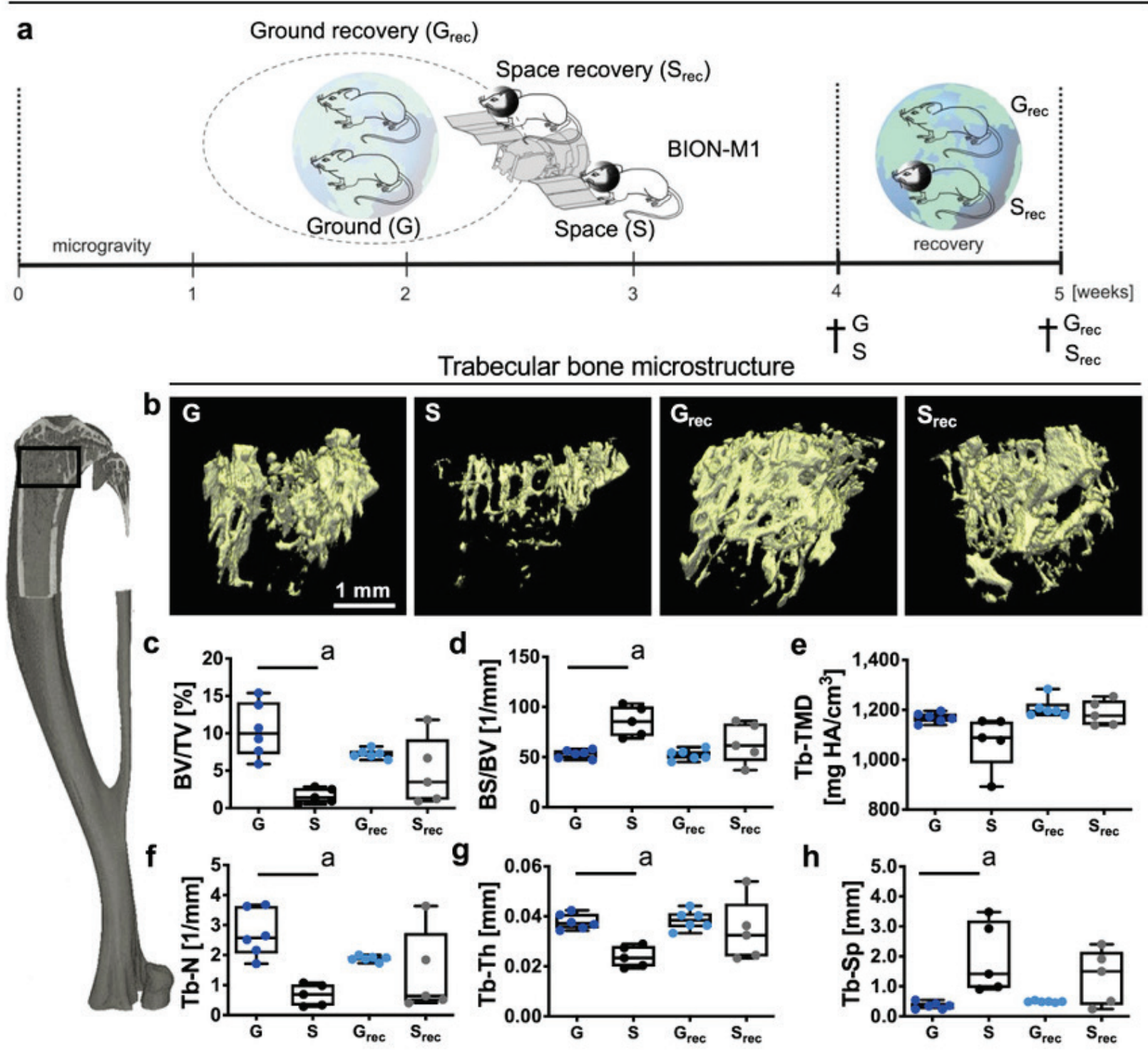
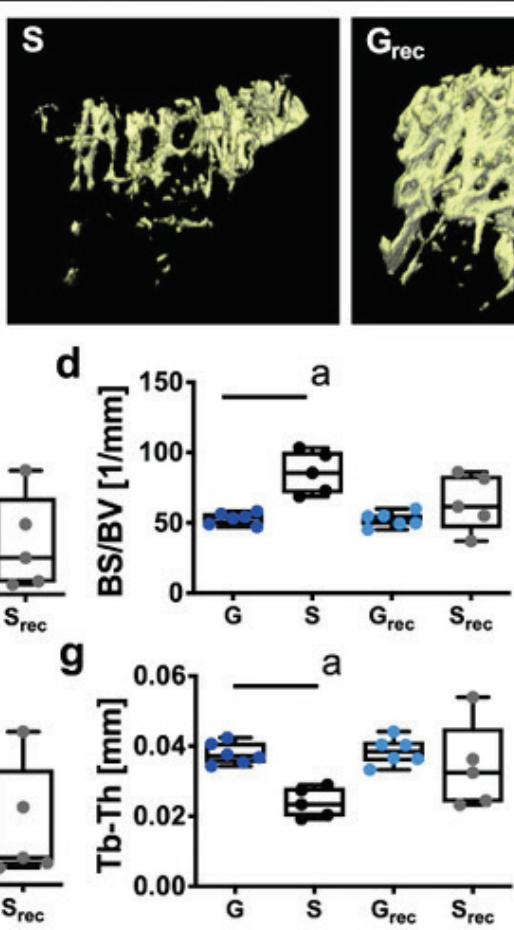
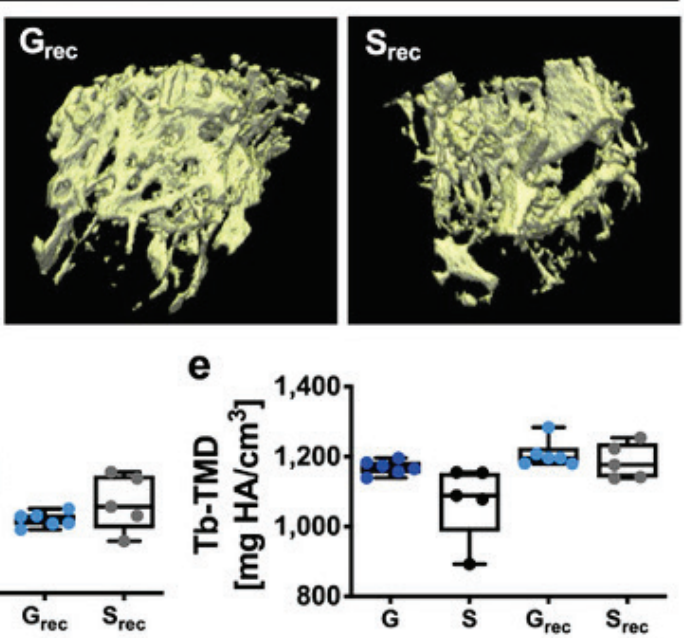

$\mathbf{h}$

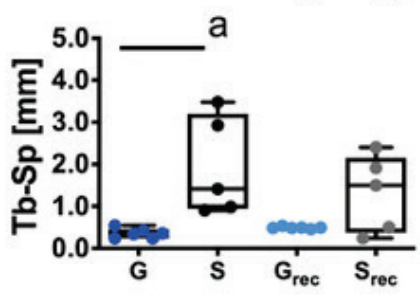

Fig. 1. $\mu \mathrm{CT}$ evaluation of $\mathrm{Tb}$ morphology showed reduced bone volume in mice after space flight and $7 \mathrm{~d}$ of recovery. (a) Study design, analysing mice that were in space for 4 weeks on the BION-M1 mission (S), followed by 1 week of recovery on Earth $\left(\mathrm{S}_{\text {rec }}\right)$, compared to control mice kept on the ground for $4(\mathrm{G})$ and 5 weeks $\left(\mathrm{G}_{\text {rec }}\right)$, respectively. (b) 3D $\mu \mathrm{CT}$ images of Tb-bone in the proximal tibia analysis revealed (c) reduced $\mathrm{Tb}-\mathrm{BV} / \mathrm{TV}$ in $\mathrm{S}$ compared to $\mathrm{G}$ and (d) a higher ratio of bone surface to bone volume (BS/BV) in $\mathrm{S}$ than in G. (e) While Tb-TMD was similar in all groups, Tb microstructural analysis showed that (f) $\mathrm{Tb}-\mathrm{N}$ and (g) $\mathrm{Tb}-\mathrm{Th}$ were significantly lower and (h) Tb-Sp was higher in S than in G. Groups: S, $n=5, \mathrm{G}, n=6, \mathrm{~S}_{\mathrm{rec}^{\prime}} n=5, \mathrm{G}_{\mathrm{rec}^{\prime}} n=6$. 
provided by the manufacturer. Objects between 100 and 2,000 $\mu^{3}$ volume were assumed to be osteocyte lacunae, while objects greater than 2,000 $\mu^{3}$ were regarded as vascular canals and objects below $100 \mu \mathrm{m}^{3}$ were excluded as noise.

\section{Osteocyte canaliculi evaluation}

A modified Ploton's silver nitrate precipitation with thionine contrast was performed on PMMA sections of the distal tibia as previously described (Derkx and Birkenhäger-Frenkel 1995; Jáuregui et al., 2016). Osteocyte canaliculi in Tb- and Ct-bone $1 \mathrm{~mm}$ proximal of the growth plate were determined using ImageJ software (NIH, University of Wisconsin, USA), considering canaliculi connected with the lacuna in the plane of view in both the trabecular $(\mathrm{Tb}-\mathrm{N}-\mathrm{Ca} / \mathrm{Lc})$ and cortical $(\mathrm{Ct}-\mathrm{N}-\mathrm{Ca} / \mathrm{Lc})$ regions 10 to 20 osteocyte lacunae were evaluated.

\section{Cellular histomorphometry}

To measure osteoclast activity, the number of TRAPpositive cells on the Tb-bone surface, based on TRAP activity, was determined by staining PMMA sections with naphthol AS-MX phosphate (Sigma-Aldrich) and Fast Red Violet LB salt (Sigma-Aldrich) in $40 \mathrm{mmol} / \mathrm{L}$ acetate buffer ( $\mathrm{pH}$ 5.0). The numbers of TRAP-positive cells per bone perimeter (TRAP ${ }^{+}$Cells/
BPm, 1/mm) and TRAP-positive cellular surface per bone surface $\left(\mathrm{TRAP}^{+}\right.$Cell-S/BS, \%) were determined 0.5-1.5 mm below the growth plate in the Tb-bone compartment.

Toluidine blue staining was performed on PMMA-embedded bone sections. Viable osteocytes in the $\mathrm{Tb}$ and $\mathrm{Ct}$ compartment of the tibia, defined as osteocyte lacunae with stained cell nuclei and osteocyte lacunae without stained cell nuclei, were assessed and presented as the ratio of empty lacunae in relation to total osteocyte lacunae (eLc, \%) using the OsteoMeasure system (OsteoMetrics, USA).

Osteocyte apoptosis was assessed by performing a TUNEL assay on the bone sections using a In Situ CellDeathDetection Kit (Roche) according to the manufacturer's instructions. The percentage of TUNEL-positive osteocytes over TUNEL-negative osteocytes (TUNEL+ Ot) in the Ct-bone and Tb-bone was determined

\section{Statistical analysis}

All analyses were done in a blinded manner. Collected data are presented as the median \pm IQR. Significant differences were determined with the Kruskal-Wallis test and subsequent Dunn's multiple comparison testing $(\alpha=0.05)$ for the comparisons ground-space, ground-ground recovery, space-space

\section{Cortical bone microstructure}
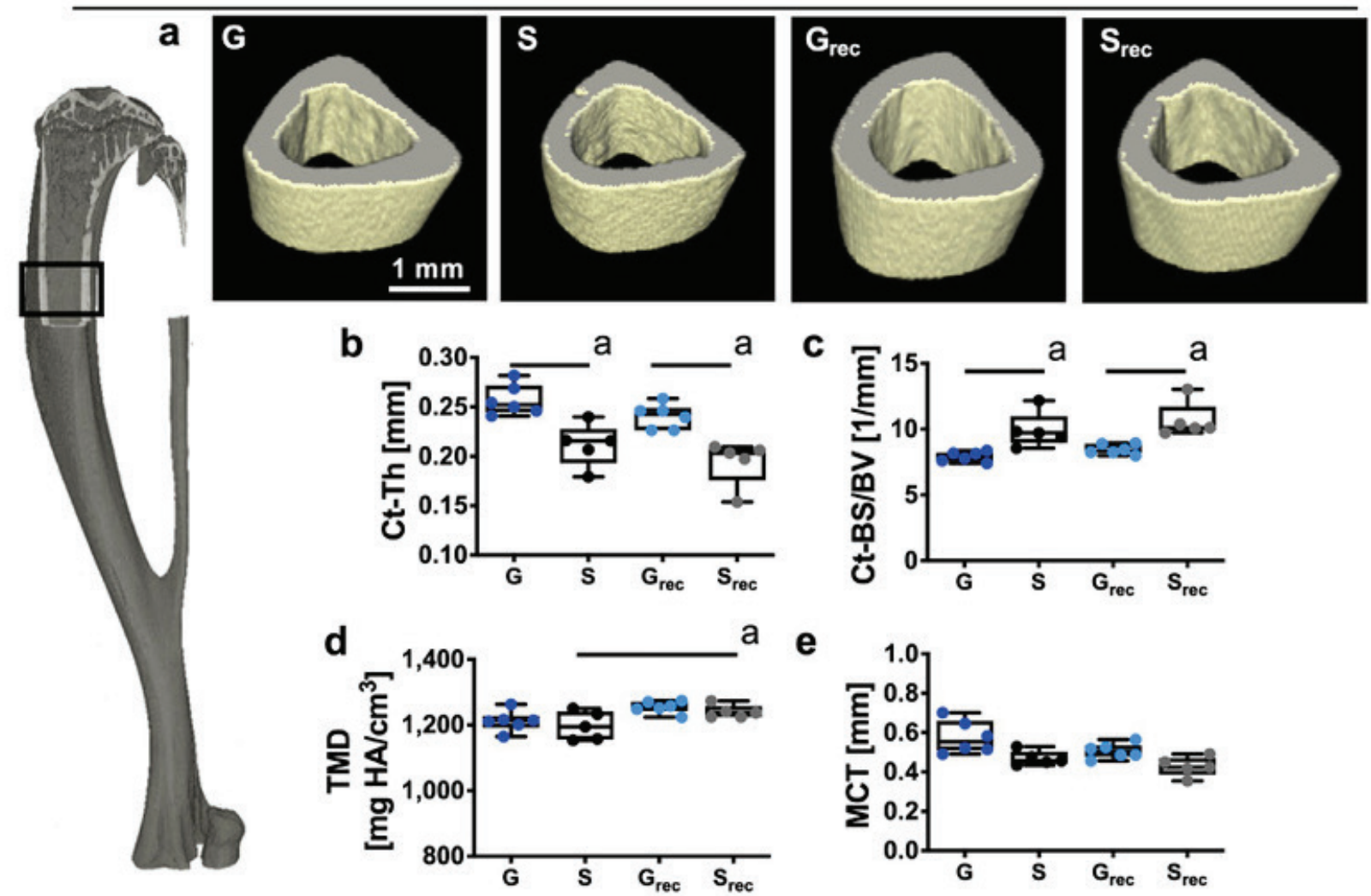

Fig. 2. Lower $\mathrm{Ct}-\mathrm{Th}$ in space is persistent after subsequent recovery. (a) $\mu \mathrm{CT}$ image analysis indicated (b) a significantly lower $\mathrm{Ct}$-Th in $\mathrm{S}$ than in $\mathrm{G}$, and in $\mathrm{S}_{\mathrm{rec}}$ than in $\mathrm{G}_{\mathrm{rec}}$, along with (c) a significantly lower Ct-BS/ BV in $S$ than in $G$, and in $S_{\text {rec }}$ than in $G_{\text {rec }}$ (d) Ct-TMD was higher in $S_{\text {rec }}$ than in $S$, while (e) $\mu C T$ showed no differences among groups. Groups: $S, n=5, \mathrm{G}, n=6, \mathrm{~S}_{\mathrm{rec}} n=5, \mathrm{G}_{\mathrm{rec}} n=6$. 
recovery, and ground recovery-space recovery with GraphPad Prism 7 software (GraphPad Software, San Diego, CA).

\section{Results}

\section{Tb-bone compartment recovered $7 \mathrm{~d}$ post space flight}

In this study, bone quality of tibiae from mice that were part of the BION-M1 mission was analysed to specify the effects of a one-month space flight and a 7-day recovery on Earth (Fig. 1a). To determine the changes in $\mathrm{Tb}$ microstructure following the space mission and recovery time, $3 \mathrm{D}$-reconstructed $\mu \mathrm{CT}$ images were analysed (Fig. 1b). A significantly lower $\mathrm{Tb}-\mathrm{BV}$ was determined in the $\mathrm{S}$ compared to the $\mathrm{G}$ group (Fig. 1c, BV/TV, $p=0.0029$ ). The bone surface was larger in the $\mathrm{S}$ group, suggesting $\mathrm{Tb}$-bone loss by surface removal (Fig. 1d, BS/BV, $p=0.0181$ ). A loss of $\mathrm{Tb}$-TMD could not be detected in the $S$ group or as a result of recovery on Earth (Fig. 1e; TMD). The observed lower Tb-BV/TV in the $S$ group was supported by a significantly lower $\mathrm{Tb}-\mathrm{N}$ and a lower $\mathrm{Tb}$-Th in $\mathrm{S}$ than on the G group (Fig. 1f, Tb-N, $p=0.005$; and Fig. 1g, Tb-Th, $p=0.018$ ). The resultant $\mathrm{Tb}$ separation was significantly higher in the $S$ compared to the G group (Fig. 1h, Tb-Sp, $p=0.004$ ).

\section{Ct-bone loss did not fully recover post space flight}

The 3D reconstruction of the $\mu \mathrm{CT}$ images showed changes in Ct-bone microstructure (Fig. 2a). Significantly lower Ct-Th was present in the $S$ group compared to ground (Fig. 2b, Ct-Th, $p=0.025$ ) and persisted in the $S_{\text {rec }}$ group compared to $\mathrm{G}_{\text {rec }}(p=0.038)$. The loss of bone matrix was further confirmed by larger $\mathrm{Ct}$ surface values in the $\mathrm{S}$ group compared to the G group (Fig. 2c, $p=0.014$ ) and in the $S_{\text {rec }}$ group compared to the $\mathrm{G}_{\text {rec }}$ group $(p=0.050)$. Ct material quality in terms of Ct-TMD was significantly elevated upon $S_{\text {rec }}$ compared to the $S$ group (Fig. $2 d, p=0.034$ ). Shape adaptations contributing to $\mathrm{Ct}$-bone loss could not be detected by the calculation of the MCT (Fig. 2e, MCT).

\section{Similar Ca contents determined by qBEI}

Hydroxyapatite minerals influence the intrinsic toughness and biomechanical properties of the bone matrix. To further investigate the mineralisation degree, qBEI was performed to determine the Ca content of the bone matrix. Representative images of Ct-bone presented with comparable mineralisation (Fig. 3a). qBEI confirmed the lack of significant alterations in mean calcium content in $\mathrm{Tb}$ - and $\mathrm{Ct}$ bone (Fig. 3b,c, CaMean).

\section{Higher TRAP-positive cell activity following space flight}

The cause of the lower BV/TV in the Tb-bone compartment due to space flight was further analysed by determining TRAP-positive bone-surface cells as a measurement of osteoclast activity (Fig. 4a). A significant elevation of TRAP-positive cells per Bpm as well as a larger TRAP-positive cellular surface per BS in space compared to ground were determined (Fig. 4b, $p=0.055$; and Fig. 4c, $p=0.037$ ). In addition, TRAP-positive osteocytes were seen within the bone matrix (Fig. 4d).

\section{Impaired LCN in Ct-bone following space flight} The LCN as a functional entity within the bone matrix is highly dependent on the number individual network characteristics. 3D reconstruction of the $\mathrm{Ct}$ shell, including the separation of blood vessel canals and osteocyte lacunae, was performed (Fig. 5a).

\section{Mineralisation degree}

a

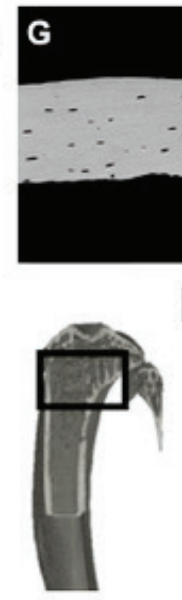

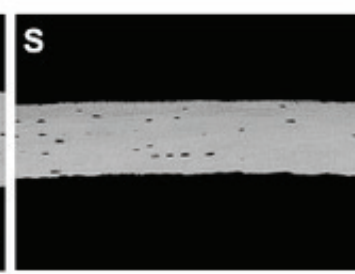

b

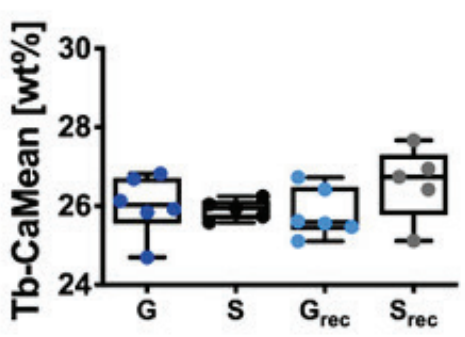

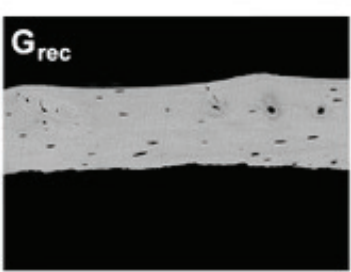

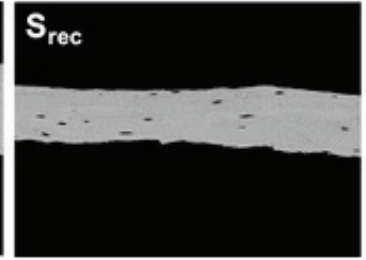

C
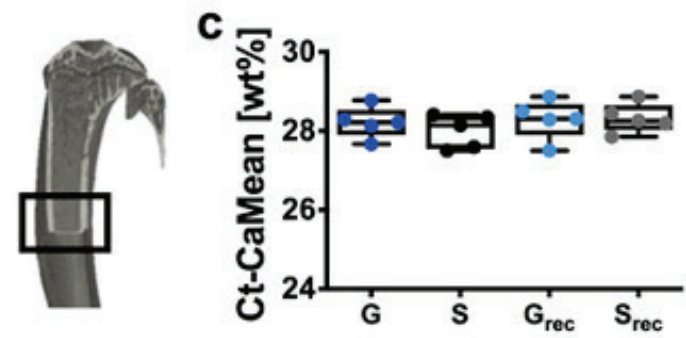

Fig. 3. Bone matrix calcium content was not affected during space flight. (a) Representative images of the Ct compartment detected by qBEI. Evaluation showed similar (b) CaMean and (c) BMDD in Tb- and (d,e) Ct-bone. Groups: $\mathrm{S}, n=5, \mathrm{G}, n=6, \mathrm{~S}_{\mathrm{rec}^{\prime}} n=5, \mathrm{G}_{\mathrm{rec}^{\prime}} n=6$. 
In both compartments, the lacunar characteristics were comparable between the groups. Osteocyte lacuna number and mean osteocyte $\mathrm{Lc}-\mathrm{V}$ were not significantly affected by space flight in $\mathrm{Tb}$ - or $\mathrm{Ct}$ bone (Fig. 5b,c, N-Lc/TV; and Fig. 5d,e, Lc-V), but also sphericity and orientation of the lacunae was unchanged (data not shown). Therefore, a measurable 3D adaptation of the lacunar characteristics could not be provided, suggesting a small contribution of lacunar remodelling during space flight and recovery. As determinants for mechanotransfer, but also for provision of nutrition and waste product removal, the canaliculi contribute to the LCN connectivity. Canaliculi number in 2D were determined using the Ploton's silver nitrate staining (Fig. 5f). While significant changes following the space mission were absent in both $\mathrm{Ct}$ - and $\mathrm{Tb}$-bone, a non-significant tendency towards fewer canaliculi per Lc was seen in $\mathrm{Ct}$-bone in the $\mathrm{S}_{\text {rec }}$ group compared to the $\mathrm{G}_{\text {rec }}$ group (Fig. 5g,h, N-Ca/Ot-Lc, Ct: $p=0.077$ ).

Cell viability is the primary element to ensure function. Osteocyte viability was determined on histological sections stained with toluidine blue. The percentage of empty osteocyte lacunae in $\mathrm{Tb}$ bone remained unchanged with space flight, while a significantly higher percentage of empty lacunae was determined in the $S$ group compared to the $G$ group (Fig. 5i, j,, $\mathbf{k}$, eLc, for Tb: $p=0.037$ ). Osteocyte apoptosis determined by a TUNEL assay showed
TUNEL+ osteocytes (Fig. 5l) with similar results for $\mathrm{Ct}$ - and $\mathrm{Tb}$-bone (Fig. 5m,n).

\section{Discussion}

The absence of mechanical loading on bone, as experienced during microgravity in space flight or long-term immobilisation, provokes rapid Ca release, leading to compositional and mechanical changes within the bone matrix. For treatment and prevention techniques as well as a further understanding of load-induced mechanisms in bone, it is essential to characterise these changes. In this study, both bone compartments, $\mathrm{Tb}$ and $\mathrm{Ct}$, showed a compromised microstructure immediately following a space mission, while only $\mathrm{Tb}$-bone volume fully recovered by $7 \mathrm{~d}$ post space flight. Whereas a reduction in $\mathrm{Tb}$ bone was also observed in previous studies (Gerbaix et al., 2017; Maupin et al., 2019), evidence for its recovery has not yet been presented. Moreover, $\mathrm{Ct}$ microstructure is even further compromised following recovery (Gerbaix et al., 2017). A recovery period following a space mission was associated with denser Ct-TMD than when in space, showing the recovery of mineralisation in the $\mathrm{Ct}$ compartment, whereas changes in Tb-TMD were absent. Based on the structural recovery solely in the Tb compartment, 2 differential processes are suggested. The results
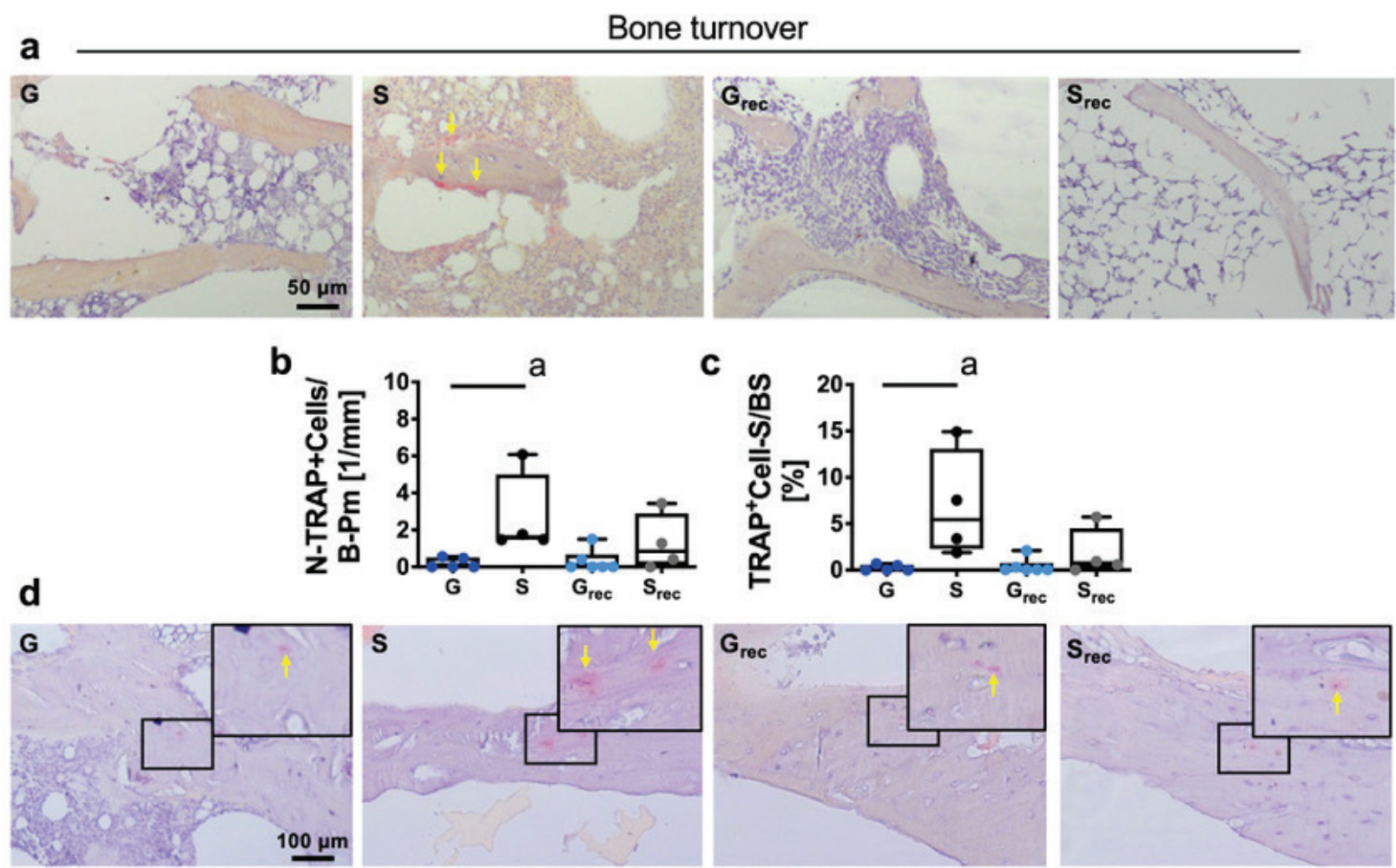

Fig. 4. TRAP-positive cell activity was higher following space flight. (a) Analysis of TRAP-stained sections in Tb-bone revealed (b) a higher number of TRAP-positive cells per bone perimeter (highlighted by yellow arrows) in $S$ than in $G$, along with (c) a higher TRAP-positive cellular surface over the bone surface in $S$ than in G. (d) TRAP staining showed the presence of TRAP-positive osteocytes. Groups: $S, n=4, \mathrm{G}, n=5$, $\mathrm{S}_{\mathrm{rec}^{\prime}} n=4, \mathrm{G}_{\text {rec }^{\prime}} n=5$. 


\section{Compartment specific osteocyte morphology}
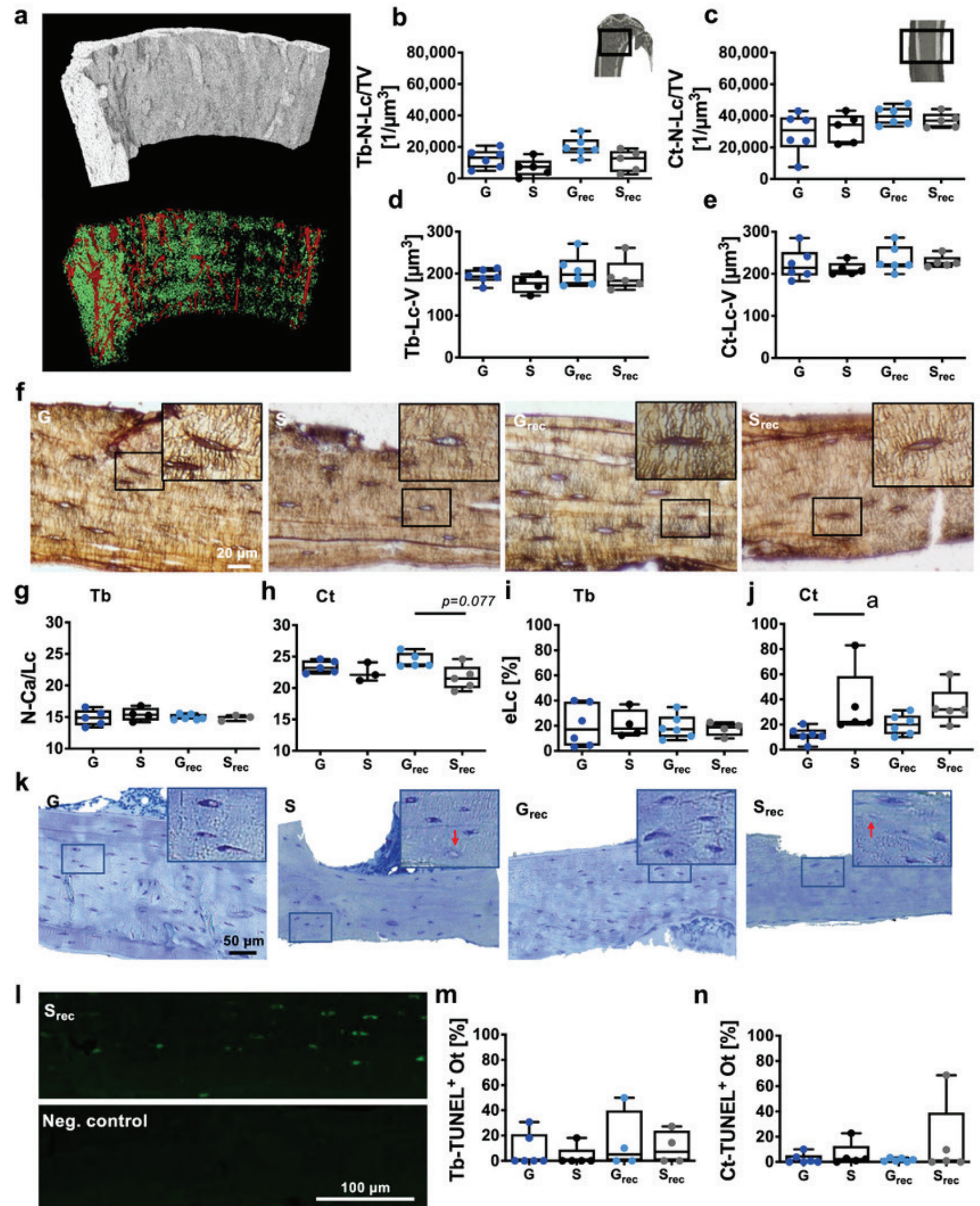

n

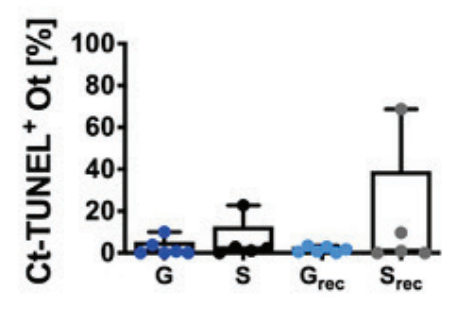

Fig. 5. Osteocyte morphology was similar in all groups for both compartments, along with changes in the number of empty osteocyte lacunae in Ct-bone. (a) 3D reconstruction of the Ct-bone with embedded blood vessel channels and osteocyte lacunae (lower panel) showing (b) a similar N-Lc/TV in Tb- and (c) Ct-bone along with $(\mathbf{d}, \mathbf{e})$ the same $\mathrm{Lc}-\mathrm{V}$ in both compartments. (f) Silver precipitation visualising the LCN revealing (g) an unchanged $\mathrm{N}-\mathrm{Ca} / \mathrm{Lc}$ in Tb-bone and (h) a tendency towards fewer $\mathrm{N}-\mathrm{Ca} / \mathrm{Lc}$ in Ct-bone of $\mathrm{S}_{\mathrm{rec}}$ compared to $G_{\text {rec }}$ (i) The percentage of eLc was similar in Tb-bone, while in (j) Ct-bone, a higher percentage was observed in the $S$ than in the G. (1) TUNEL assay to determine local rate of osteocyte apoptosis. TUNEL+ Ot are visible in the $\mathrm{Ct}$ bone of one representative section from the Srec group. Negative staining control did not show TUNEL+ cells. (m) In Ct and (n) Tb bone no significant differences of TUNEL+ Ot were determined. Groups: $\mathrm{S}, n=4, \mathrm{G}, n=5, \mathrm{~S}_{\mathrm{rec}^{\prime}} n=4, \mathrm{G}_{\mathrm{rec}} n=5$. 
point to a larger bone surface area due to resorption immediately following the space mission, which might contribute to the rebuilding of $\mathrm{Tb}$-bone on ground. The metabolically less-active Ct-bone structure had not yet recovered, pointing towards a recovery of TMD in Ct-bone that was independent of bone volume, reflecting compositional changes in the bone matrix. Others do not find TMD differences in $\mathrm{Tb}$ - or Ct-bone when subjected to space flight (Gerbaix et al., 2017), whereas the current study's results confirmed the observed microgravity-induced bone loss of individuals travelling to space (Orwoll et al., 2013). Additionally, further qBEI analysis of regional BMDD showed no significant differences in either compartment. Bone mineral density analyses obtained using $\mu \mathrm{CT}$ were performed on a volumetric and larger region of interest with calibration based on hydroxyapatite phantoms, whereas areal qBEI data were obtained at a higher resolution based on $\mathrm{Ca}$ and $\mathrm{Al}$ standard calibration.

Bone loss is initiated by imbalanced bone turnover, which was seen in Tb-bone as a higher TRAP-positive cell number, an indication of greater osteoclast activity in the $S$ group than in the G group. Osteocytic osteolysis has been reported by Blaber et al. (2013). Enlarged osteocyte lacunae are reported to respond more strongly to mechanical loading (Hemmatian et al., 2018). However, when TRAPpositive osteocytes were examined, no differences in 3D osteocyte lacunar morphology were found within the groups and compartments, indicating the absence of significantly higher osteocytic osteolysis activity following space flight.

Bone remodelling is driven by the regulating action of the mechanosensitive osteocyte network (Prideaux et al., 2016), a well-connected LCN, which is essential for osteocyte-mediated bone turnover. It was found that osteocytes in Ct-bone alone present with a non-significant trend towards fewer canaliculi in the $S_{\text {rec }}$ group than in the $G_{\text {rec }}$ group, implicating an impairment of the LCN with potential effects on Ct-bone recovery. A well-connected LCN is essential for osteocyte-mediated signalling and is reduced in Ct-bone following unloading (Rolvien et al., 2020). An age-related decrease in osteocyte canaliculi independent of mechanical loading has been shown and linked to the mechanosensitivity of osteocytes (Milovanovic et al., 2013). One fate of osteocytes is cell death, e.g. by apoptosis or necrosis, which might occur due to inadequate mechanical loading resulting in an empty osteocyte lacuna within the bone matrix. While in Tb-bone an unchanged percentage of empty lacunae was apparent, Ct-bone presented with a significantly higher proportion of empty lacunae in the $S$ group. In the past, no signs of osteocyte cell death have been detected during a $15 \mathrm{~d}$ mission (Blaber et al., 2013), which raises the assumption that osteocyte cell death due to microgravity depends on the exposure time, which is supported by a study analysing bone after a $30 \mathrm{~d}$ space mission and $8 \mathrm{~d}$ recovery (Gerbaix et al., 2017). Since Ct-bone remodelling following space flight is insufficient, the physiological process of dying osteocytes is not counterbalanced, leading to an accumulation of empty osteocyte lacunae without replacement by newly embedded osteoblasts.

While animals traveling to space are rather unusual and experiments are seldom performed, this study had certain limitations. The material obtained from mice following space flight was limited in number, and focused on only one skeletal element, i.e. the tibia. In some samples, the evaluation of the Tb-bone was challenging, reducing the group size for some methods to guarantee high-quality analysis. Furthermore, the absence of $\mathrm{Ct}$-bone recovery might

\section{Effects on bone compartments in microgravity and recovery}

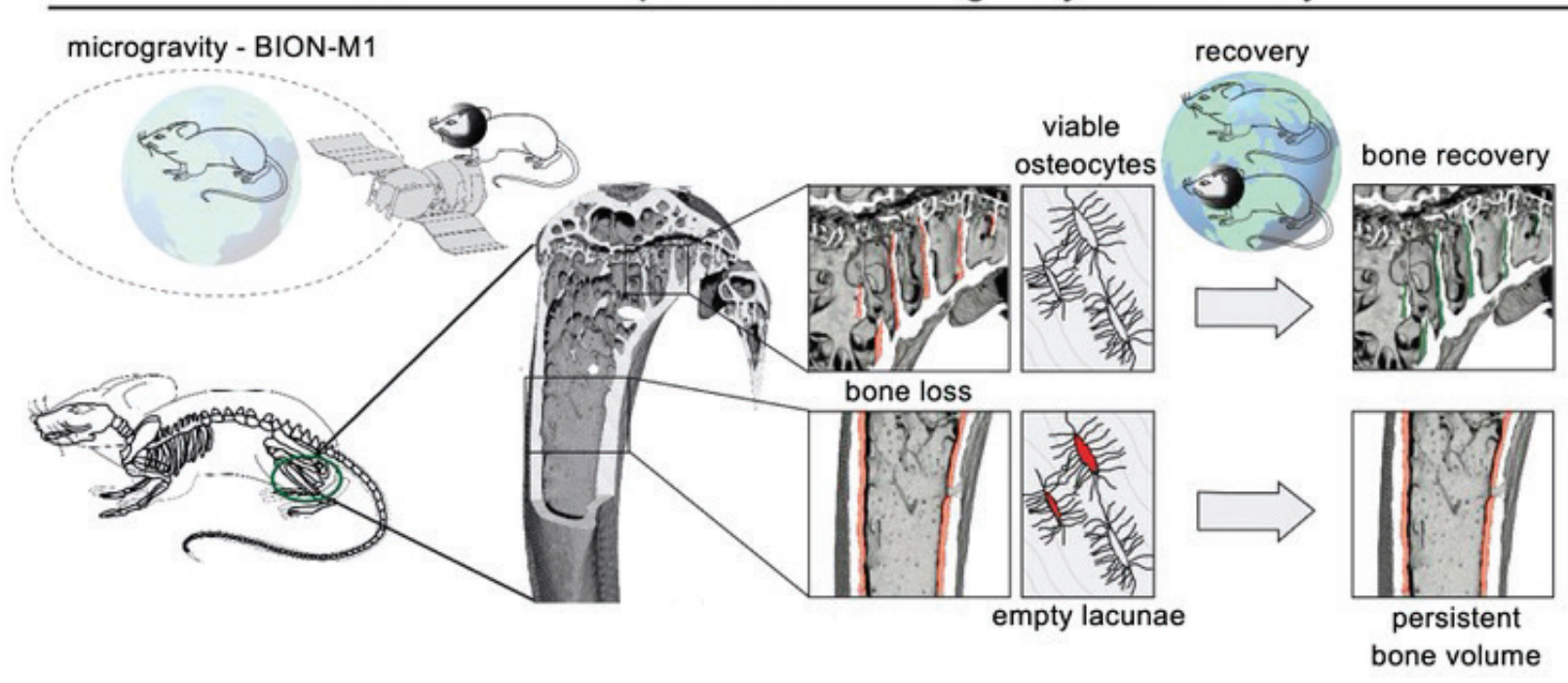

Fig. 6. Differential effects were observed in bone compartments following microgravity and recovery. Overall, the analysis revealed $\mathrm{Tb}$ - and Ct-bone loss, which was accompanied by more empty osteocyte lacunae in Ct-bone. While Tb-bone recovered during a recovery period of $7 \mathrm{~d}$, in Ct-bone, the lower bone volume persisted. 
be an effect of a compartment-specific prolonged recovery period, which has been postulated for human bone following space flight (Smith et al., 2005). While only a relatively short recovery period of $7 \mathrm{~d}$ was analysed, the aim was to determine skeletalspecific changes directly following space flight in order to provide further data for possible treatment options to recover bone loss in astronauts.

\section{Conclusions}

In summary, the supposition that microgravity induces bone loss in $\mathrm{Tb}$ - and $\mathrm{Ct}$-bone was confirmed while providing new information demonstrating that $\mathrm{Tb}$-bone loss recovers, whereas Ct-bone loss persists. Importantly, the assessment of osteocyte viability and morphology identified reduced osteocyte viability solely in Ct-bone without compensatory mechanisms being initiated, while osteocyte canaliculi showed no significant difference, a tendency was noted towards slightly fewer osteocyte canaliculi in the $S_{\text {rec }}$ compared to the $S$ group, suggesting an impaired LCN, endangering mechano-dependent bone remodelling and thereby changing the properties of the bone matrix. The observations of differential responses to microgravity and recovery in $\mathrm{Tb}$ - and $\mathrm{Ct}$-bone could be linked to different dimensions of osteocyte conservation in both compartments (Fig. 6).

Tb-bone is more accessible to new bone formation upon loading due to a more preserved osteocyte quality. Further studies are needed to validate the contribution of unloading-induced osteocyte pathology to bone material quality following space flight. The observed changes to Ct-bone osteocytes did not recover within $7 \mathrm{~d}$, potentially leading to a manifested change in Ct-bone quality due to microgravity. However, the validation of the contribution of the unloading-induced osteocyte pathology to bone material quality following space flight requires further investigation (i) to identify the underlying mechanisms leading to differential responses of $\mathrm{Tb}$ - and $\mathrm{Ct}$-bone under unloading, (ii) to determine whether $\mathrm{Ct}$-bone recovery is prolonged or absent, and (iii) to identify osteocyte apoptosis prevention strategies to obviate irreversible bone loss during space flight and as an approach to treat agerelated osteocyte death. These investigations should aim to better understand unloading-induced bone loss to optimise prevention and treatment options for astronauts and patients with immobilisation and other bone diseases.

\section{Acknowledgments}

Mona Neven, Olga Winter and Andrea Thieke are thanked for technical assistance.

K. Jähn-Rickert is supported by the German Research Foundation (DFG) under grant no. JA 2654/1-1 and is awarded with the 2018 IFRMS fellowship grant. B. Busse is supported by the German Research Foundation (DFG) under grant no. BU 2562/3-1. T. Rolvien is supported by the German Research Foundation (DFG) under grant \# RO 5925/11.

All authors declare no competing interests.

\section{Author contributions}

Simon von Kroge: data collection, data analysis, manuscript preparation and editing. Eva M. Wölfel: data collection, data analysis, manuscript preparation and editing. Lyudmila Buravkova: planning, data collection, manuscript preparation. Dmitri A. Atiakshin: planning, data collection, manuscript preparation. Elena Markina: planning, data collection, manuscript preparation. Thorsten Schinke: data analysis, manuscript preparation and editing. Tim Rolvien: data collection, data analysis, manuscript preparation and editing. Björn Busse: planning, data analysis, manuscript preparation and editing. Katharina Jähn-Rickert: planning, data collection, data analysis, manuscript preparation and editing.

\section{References}

Aguirre JI, Plotkin LI, Stewart SA, Weinstein RS, Parfitt AM, Manolagas SC, Bellido T (2006) Osteocyte apoptosis is induced by weightlessness in mice and precedes osteoclast recruitment and bone loss. J Bone Miner Res 21: 605-615.

Allen MR, Burr DB (2014) Bone modeling and remodeling. Basic and applied bone biology, Elsevier. Chapter 4. pp 75-90.

Andreev-Andrievskiy A, Popova A, Boyle R, Alberts J, Shenkman B, Vinogradova O, Dolgov O, Anokhin K, Tsvirkun D, Soldatov P, Nemirovskaya T, Ilyin E, Sychev V (2014) Mice in Bion-M 1 space mission: training and selection. PLoS One 9: e104830. DOI: 10.1371/journal.pone.0104830.

Andreev-Andrievsky AA, Shenkman BS, Popova AS, Dolguikh ON, Anokhin KV, Soldatov PE, Ilyin EA, Sychev VN (2014) [Experimental studies with mice on the program of the biosatellite BION-M1 mission]. Aviakosm Ekolog Med 48: 14-27.

Blaber EA, Dvorochkin N, Lee C, S. Alwood JS, Yousuf R, Pianetta P, Globus RK, Burns BP, Almeida EAC (2013) Microgravity induces pelvic bone loss through osteoclastic activity, osteocytic osteolysis, and osteoblastic cell cycle inhibition by CDKN1a/ P21. PLoS One 8: e61372. DOI: 10.1371/journal. pone.0061372.

Bouxsein ML, Boyd SK, Christiansen BA, Guldberg RE, Jepsen KJ, Müller R (2010) Guidelines for assessment of bone microstructure in rodents using micro-computed tomography. J Bone Miner Res 25: 1468-1486.

Buravkova LB, Gershovich PM, Gershovich JG, Grigorev AI (2010) Mechanisms of gravitational 
sensitivity of osteogenic precursor cells. Acta Naturae 2: 28-36.

Busse B, Hahn M, Soltau M, Zustin J, Püschel K, Duda GN, Amling M (2009) Increased calcium content and inhomogeneity of mineralization render bone toughness in osteoporosis: mineralization, morphology and biomechanics of human single trabeculae. Bone 45: 1034-1043.

Caillot-Augusseau A, Lafage-Proust $\mathrm{MH}$, Soler C, Pernod J, Dubois F, Alexandre C (1998) Bone formation and resorption biological markers in cosmonauts during and after a 180-day space flight (Euromir 95). Clin Chem 44: 578-585.

Collet P, Uebelhart D, Vico L, Moro L, Hartmann D, Roth M, Alexandre C (1997) Effects of 1- and 6-month spaceflight on bone mass and biochemistry in two humans. Bone 20: 547-551.

Derkx P, Birkenhäger-Frenkel DH (1995) A thionin stain for visualizing bone cells, mineralizing fronts and cement lines in undecalcified bone sections. Biotech Histochem 70: 70-74.

Gerbaix M, Gnyubkin V, Farlay D, Olivier C, Ammann P, Courbon G, Laroche N, Genthial R, Follet H, Peyrin F, Shenkman B, Gauquelin-Koch G, Vico L (2017) One-month spaceflight compromises the bone microstructure, tissue-level mechanical properties, osteocyte survival and lacunae volume in mature mice skeletons. Sci Rep 7: 2659. DOI: 10.1038/s41598017-03014-2.

Grimm D, Grosse J, Wehland M, Mann V, Reseland JE, Sundaresan A, Corydon TJ (2016) The impact of microgravity on bone in humans. Bone 87: 44-56.

Hargens AR, Vico L (2016) Long-duration bed rest as an analog to microgravity. J Appl Physiol 120: 891-903.

Hemmatian H, Laurent MR, Ghazanfari S, Vanderschueren D, Bakker AD, Klein-Nulend J, van Lenthe GH (2017) Accuracy and reproducibility of mouse cortical bone microporosity as quantified by desktop microcomputed tomography. PLoS One 12: e0182996. DOI: 10.1371/journal.pone.0182996.

Hemmatian H, Jalali R, Semeins CM, Hogervorst JMA, van Lenthe GH, Klein-Nulend J, Bakker AD (2018) Mechanical loading differentially affects osteocytes in fibulae from lactating mice compared to osteocytes in virgin mice: possible role for lacuna size. Calcif Tissue Int 103: 675-685.

Jähn K, Kelkar S, Zhao H, Xie Y, Tiede-Lewis LAM, Dusevich V, L. Dallas SL, Bonewald LF (2017) Osteocytes acidify their microenvironment in response to PTHrP in vitro and in lactating mice in vivo. J Bone Miner Res 32: 1761-1772.

Jáuregui EJ, Akil O, Acevedo C, Hall-Glenn F, S. Tsai BS, Bale HA, Liebenberg E, Humphrey MB, Ritchie RO, Lustig LR, Alliston T (2016) Parallel mechanisms suppress cochlear bone remodeling to protect hearing. Bone 89: 7-15.

Jilka RL (2013) The relevance of mouse models for investigating age-related bone loss in humans. J Gerontol A Biol Sci Med Sci 68: 1209-1217.
Leblanc AD, Schneider VS, Evans HJ, Engelbretson DA, Krebs JM (1990) Bone mineral loss and recovery after 17 weeks of bed rest. J Bone Miner Res 5: 843-850.

Maupin KA, Childress P, Brinker A, Khan F, Abeysekera I, Aguilar IN, Olivos DJ, Adam G, Savaglio MK, Ganesh V, Gorden R, Mannfeld R, Beckner E, Horan DJ, Robling AG, Chakraborty N, arti Gautam A, Hammamieh R, Kacena MA (2019) Skeletal adaptations in young male mice after 4 weeks aboard the international space station. NPJ Microgravity 5: 21. DOI: 10.1038/s41526-019-0081-4.

Milovanovic P, Zimmermann EA, Hahn M, Djonic D, Püschel K, Djuric M, Amling M, Busse B (2013) Osteocytic canalicular networks: morphological implications for altered mechanosensitivity. ACS Nano 7: 7542-7551.

Orwoll ES, Adler RA, Amin S, Binkley N, Lewiecki EM, Petak SM, Shapses SA, Sinaki M, Watts NB, Sibonga JD (2013) Skeletal health in long-duration astronauts: nature, assessment, and management recommendations from the NASA bone summit. J Bone Miner Res 28: 1243-1255.

Prideaux M, Findlay DM, Atkins GJ (2016) Osteocytes: the master cells in bone remodelling. Curr Opin Pharmacol 28: 24-30.

Rolvien T, Krause M, Jeschke A, YorganT, Püschel K, Schinke T, Busse B, Demay MB, Amling M (2017) Vitamin D regulates osteocyte survival and perilacunar remodeling in human and murine bone. Bone 103: 78-87.

Rolvien T, Milovanovic P, Schmidt FN, von Kroge S, Wölfel EM, Krause M, Wulff B, Püschel K, Ritchie RO, Amling M, Busse B (2020) Long-term immobilization in elderly females causes a specific pattern of cortical bone and osteocyte deterioration different from postmenopausal osteoporosis. J Bone Miner Res 35: 1343-1351.

Rolvien T, Schmidt T, Schmidt FN, von Kroge S, Busse B, Amling M, and Barvencik F (2019) Recovery of bone mineralization and quality during asfotase alfa treatment in an adult patient with infantile-onset hypophosphatasia. Bone 127: 67-74.

Sibonga JD, Evans HJ, Sung HG, Spector HD, Lang TF, Oganov VS, Bakulin AV, Shackelford LC, LeBlanc AD (2007) Recovery of spaceflight-induced bone loss: bone mineral density after long-duration missions as fitted with an exponential function. Bone 41: 973-978.

Smith SM, Abrams SA, Davis-Street JE, Heer M, O'Brien KO, Wastney ME, Zwart S R (2014) Fifty years of human space travel: implications for bone and calcium research. Annu Rev Nutr 34: 377-400.

Smith SM, Wastney ME, Morukov BV, Larina IM, Nyquist LE, Abrams SA, Taran EN, Shih CY, Nillen JL, Davis-Street JE, Rice BL, Lane HW (1999) Calcium metabolism before, during, and after a 3-mo spaceflight: kinetic and biochemical changes. Am J Physiol 277: 1-10.

Smith SM, Wastney ME, O’Brien KO, Morukov BV, Larina IM, Abrams SA, Davis-Street JE, Oganov V, Shackelford LC. (2005) Bone markers, calcium 
metabolism, and calcium kinetics during extendedduration space flight on the mir space station. J Bone Miner Res 20: 208-218.

Sotnezova EV, Markina EA, Andreeva ER, Buravkova LB. (2017) Myeloid precursors in the bone marrow of mice after a 30-day space mission on a Bion-M1 biosatellite. Bull Exp Biol Med 162: 496-500.

Spatz JM, Wein MN, Gooi JH, Qu Y, Garr JL, Liu S, Barry KJ, Uda Y, Lai F, Dedic C, Balcells-Camps M, Kronenberg HM, Philip Babij P, Pajevic PD (2015) The Wnt inhibitor sclerostin is up-regulated by mechanical unloading in osteocytes in vitro. J Biol Chem 290: 16744-16758.

Stein TP (2013) Weight, muscle and bone loss during space flight: another perspective. Eur J Appl Physiol 113: 2171-2181.

Vico L, Collet P, Guignandon A, Lafage-Proust MH, Thomas T, Rehailia M, Alexandre C (2000) Effects of long-term microgravity exposure on cancellous and cortical weight-bearing bones of cosmonauts. Lancet 355: 1607-1611.

Zimmermann EA, Köhne T, Bale HA, Bran Panganiban B, Gludovatz B, Zustin J, Hahn M, Amling M, Ritchie RO, Björn Busse B (2015) Modifications to nano- and microstructural quality and the effects on mechanical integrity in Paget's disease of bone. J Bone Miner Res 30: 264-273.

\section{Discussion with Reviewer}

Wing Hoi Cheung: The study revealed that microgravity induced changes in cortical bone was in comparison to trabecular bone not reversible within a one-week recovery period. Would these changes be reversible in long term and which measures could be taken to prevent and/or rescue reduced osteocyte viability?

Authors: Within the proposed murine space flight model we determined a lack of cortical bone mass recovery in the tibial midshaft region, while the cortical tissue mineral density recovered 1 week following a 4 week mission in microgravity. In addition, the trabecular compartment of the loadbearing tibia presented overall with a structural recovery; however, one can note the high variability among individual values i.e. for BV/TV. Similarly, reports on astronauts and cosmonauts determined remarkably individual differences in bone responses (Sibonga et al., 2007). Here, the aBMD measurements at the lumbar spine, mostly entailing trabecular bone, showed in general a recovery with a half life of $151 \mathrm{~d}$, while the change in aBMD values in the femoral neck, entailing cortical bone, presented with a half life of $211 \mathrm{~d}$ and presented with individuals not regaining their initial bone mass even 2 years post long-duration space missions. While, the DXA measurements on aBMD might not be optimal to evaluate bone quality and skeletal risks related to microgravity missions (Orwoll et al., 2013), the data indicate that i) the cortical bone compartment might need a prolonged period of recovery compared to the trabecular bone compartment and ii) there are quite some individual differences in skeletal response to reambulation.

Data from the current study suggested a role for osteocytes, especially their viability, in the process of skeletal recovery from microgravity. Osteocytes are central regulators of bone remodelling (Prideaux et al., 2016). Osteocyte apoptosis and necrosis have previously been reported to coincide with skeletal pathologies (Noble et al., 1997 additional reference). While the RANKL-containing apoptotic bodies from osteocytes are seen to induce bone resorption events e.g. upon microcracking (Kennedy et al., 2017 - additional reference), the impaired network connectivity might interfere with the bone formation reaction. To determine bone quality, including osteocyte characteristics with microgravity investigations and recovery from it would be of upmost importance. That optimised exercise regimens, as specified for astronauts during space missions, could be a possible intervention to sufficiently reduce bone loss is a current research focus (Orwoll et al., 2013). The pharmaceutical application of bisphosphonates administered in combination with exercise prescriptions has also been shown to reduce compartmental loss in trabecular and cortical bone during spaceflight (LeBlanc et al., 2013 - additional reference). Bisphosphonates preserve osteocyte viability (Bellido and Plotkin, 2011 - additional reference, in addition to their anti-resorptive activity. Also, drug treatment with sclerostin antibodies are a possible alternative, while potential effects on osteocytes are still under investigation urging for further research.

\section{Additional References}

Bellido T and Plotkin LI (2011) Novel actions of bisphosphonates in bone: preservation of osteoblast and osteocyte viability. Bone 49: 50-55.

Kennedy OD, Lendhey M, Mauer P, Philip A, Basta-Pljakic J, Schaffler MB (2017) Microdamage induced by in vivo reference point indentation in mice is repaired by osteocyte-apoptosis mediated remodeling. Bone 95: 192-198.

LeBlanc A, Matsumoto T, Jones J, Shapiro J, Lang T, Shackelford L, Smith SM, Evans H, Spector E, Ploutz-Snyder R, Sibonga J, Keyak J, Nakamura T, Kohri K, Ohshima O (2013) Bisphosphonates as a supplement to exercise to protect bone during longduration spaceflight. Osteoporos Int 24: 2105-2114.

Noble BS, Stevens H, Loveridge N, Reeve J (1997) Identification of apoptotic changes in osteocytes in normal and pathological human bone. Bone 20: 273282.

Editor's note: The Scientific Editor responsible for this paper was Martin Stoddart. 